\title{
Ubiquitous Angles in Equiangular Sets of Lines*
}

\author{
B. Guiduli ${ }^{1}$ and M. Rosenfeld ${ }^{2}$ \\ ${ }^{1}$ Department of Computer Science, University of Chicago, \\ 1100 E 58th Street, Chicago, IL 60637, USA \\ bdg@cs.uchicago.edu \\ ${ }^{2}$ Department of Computer Science, Pacific Lutheran University, \\ Tacoma, WA 98447, USA \\ rosenfm@plu.edu
}

\begin{abstract}
A set of lines through the origin in $\mathbf{R}^{d}$ is called equiangular if all pairs intersect in the same angle. For a given dimension $d$, there are finitely many nonisometric configurations of $(d+1)$ equiangular lines in $\mathbf{R}^{d}$. In particular, there are finitely many possible angles. Such angles can occur in only few dimensions or in infinitely many dimensions. We show that there are infinitely many ubiquitous angles $\theta$ occurring among sets of equiangular lines, i.e., for all $d$ large enough, there are $d+1 \theta$-equiangular lines in $\mathbf{R}^{d}$. We also show that there are infinitely many frequent angles, those which occur for infinitely many dimensions $d$, but are not ubiquitous.
\end{abstract}

\section{Preface (by M.R.)}

Branko instilled in us, his students, the love for geometry. He taught us to expect surprises, to follow our intuition but never to trust it fully. So while we enjoyed the wonderful journeys in Geometry Land guided by Branko, occasionally we slid down its sharp razor edge. My interest in equiangular lines is a case in point. It started in the summer of 1991 when Micha Perles was visiting us in Seattle. Branko, Micha, and I walked together on a (rare) sunny day. I asked them the following question: the four diagonals of the regular cube form four equiangular lines in $\mathbf{R}^{3}$. The six diagonals of the regular icosahedron form six equiangular lines. My intuition (mis)led me to suggest that if one removes two of the six diagonals of the icosahedron, one could somehow open the remaining four diagonals so that they remain equiangular until they "match" the four diagonals of the cube. More specifically, I suggested that there are infinitely many angles among four

\footnotetext{
* The research of the second author was supported in part by NSF Grant INT-9802416.
} 
equiangular lines in $\mathbf{R}^{3}$. Micha immediately countered that he believed that the measure of the set of distinct angles will be zero. Branko suggested we try to resolve the question. The following day, I told Branko that I found a proof that not only was I wrong and Micha right, but there are only finitely many angles among four equiangular lines in $\mathbf{R}^{3}$. At that point Branko insisted that I should find out exactly how many. To my surprise, I found that there are exactly two possible angles: $\arccos \left(\frac{1}{3}\right)$ and $\arccos (1 / \sqrt{5})$ [R2], [R1]. I was quite surprised that we had not noticed this elementary fact before, and of course willing to admit that my geometric intuition once again misled me. Thus, again, Branko's "push and shove" led me to explore the fascinating world of equiangular lines developed primarily by Seidel.

\section{Introduction}

The elliptic space $\mathcal{E}^{d-1}$ is the metric space whose points are lines through the origin in $\mathbf{R}^{d}$. The distance between two points is the (smaller) angle $\theta$ between the lines $(0 \leq \theta \leq \pi / 2)$. A set $\left\{\ell_{1}, \ell_{2}, \ldots, \ell_{n}\right\}$ of such lines is $\theta$-equiangular if the angle between every pair of lines is $\theta$. In the elliptic space setting, this is an equilateral set of points in the metric space $\mathcal{E}^{d-1}$.

The study of equiangular lines in $\mathbf{R}^{d}$ was primarily motivated by Seidel's pursuit of the congruence order of $\mathcal{E}^{d-1}$. In 1947 Haantjes and Seidel [HS] proved that the congruence order of $\mathcal{E}^{2}$ is 7 . This means that if $\mathcal{M}$ is a metric space such that every subset of seven points from $\mathcal{M}$ can be isometrically embedded in $\mathcal{E}^{2}$, then $\mathcal{M}$ can be isometrically embedded in $\mathcal{E}^{2}$. Seidel conjectured (still open) that the congruence order of $\mathcal{E}^{d-1}$ is $v(d)+1$ where $v(d)$ is the maximum number of equiangular lines in $\mathbf{R}^{d}$. He then took us on a wonderful, exciting journey in search of equiangular lines. The search involved numerous interactions with many mathematical areas, intricate constructions, and many surprising results. Already in $\mathcal{E}^{2}$ we can find "counterintuitive" phenomena. For instance, if you take six lines through the origin in $\mathbf{R}^{3}$ (6 points in $\mathcal{E}^{2}$ ), then the smallest angle among the 30 angles determined by them is at most $\arccos (1 / \sqrt{5})$ and the six diagonals of the regular icosahedron attain this bound. "Intuitively" if we remove one diagonal, one could expect to be able to spread the remaining five diagonals a bit further so that the smallest angle among them will be slightly greater than $\arccos (1 / \sqrt{5})$. $\mathrm{N}$. Sloane (private communication) assured us that this indeed is not the case. That is the smallest distance among five points in $\mathcal{E}^{2}$ never exceeds $\arccos (1 / \sqrt{5})$.

The unpredictable behavior of the function $v(d)$ suggests that the metric space $\mathcal{E}^{d-1}$ is packed with "wild" structures that may be applicable to solve old geometric conjectures or, more likely,"destroy" them. Indeed, this was the case of the Borsuk Conjecture. Counterexamples to Borsuk's conjecture were constructed by Kahn and Kalai [KK] using cover properties of $\mathcal{E}^{d-1}$. For a proof from THE BOOK see [AZ].

In this note, we look for some "expected surprises," unexpected phenomena in $\mathcal{E}^{d-1}$. We hope to find them among "ubiquitous" angles. We study angles among $d+1$ equiangular lines in $\mathbf{R}^{d}$ that occur in infinitely many dimensions. We distinguish two types of such angles: ubiquitous angles, those that occur in all but finitely many dimensions, and frequent angles, those that occur in infinitely many dimensions but are not ubiquitous (yes! they exist). 
The relation between $\theta$-equiangular lines in $\mathbf{R}^{d}$ and graphs dates back to works of Blumenthal [B] and van Lint and Seidel [vLS]. To a set of $n$ equiangular lines we associate a graph $G$ as follows: on each line we select a unit vector (choose the direction arbitrarily); these represent the vertices of the graph. We connect two vertices by an edge if the angle between the two corresponding vectors is obtuse. Let $V=\left\{v_{1}, v_{2}, \ldots, v_{n}\right\}$ $\left(\ell_{i}=\left\langle v_{i}\right\rangle\right)$ be the vectors, set $\alpha=\cos \theta$, and let $d$ be the dimension of the subspace spanned by the set of lines. Let $G=\left(g_{i j}\right)$ be the Gram matrix, $g_{i j}=v_{i}^{t} v_{j}$, and let $S$ be the symmetric \pm 1 matrix defined by $S=(1 / \alpha)(G-I)$. These matrices have the form

$$
G=\left(\begin{array}{ccc}
1 & & \pm \alpha \\
& \ddots & \\
\pm \alpha & & 1
\end{array}\right), \quad S=\left(\begin{array}{ccc}
0 & & \pm 1 \\
& \ddots & \\
\pm 1 & & 0
\end{array}\right)
$$

A symmetric matrix $S$ in which the diagonal entries are zero and all other entries are \pm 1 is an $S$-matrix. Note that $G$ is positive semidefinite with smallest eigenvalue 0 of multiplicity $n-d$ (to avoid trivialities, we always assume that $n>d$ ). Consequently, $S$ has smallest eigenvalue $-1 / \alpha$ with the same multiplicity $n-d$. While the matrices $S$ and $G$ depend on the choice of the vectors $v_{i}$, the spectra of $G$ and $S$ are independent of these choices. The relation between the various graphs that can be obtained from a given set of $n \theta$-equiangular lines in $\mathbf{R}^{d}$ is an equivalence relation, they belong to the same switching class.

Definition. Switching a graph $G$ on a subset $A$ of the vertex set, is the operation of reversing all of the adjacencies between vertices in $A$ and vertices in $V(G) \backslash A$ (see [S]).

The process described above is reversible. That is from any given S-matrix $S$ we can construct the positive semidefinite matrix $G$ and thus construct $n$ equiangular lines in $\mathbf{R}^{d}\left(d=n-\right.$ multiplicity $\sigma_{\min }(S)$, where $\sigma_{\min }(S)$ is the smallest eigenvalue of $\left.S\right)$. This fundamental relation, summarized below, is the foundation for much of the study of equiangular lines [B], [vLS].

Theorem 2.1 (Blumenthal, van Lint, Seidel). There is a one-to-one correspondence between isometric classes of sets of equiangular lines and isomorphism classes of switching classes of graphs.

Thus the problem of determining which angles arise as angles of nondegenerate equiangular lines is reduced to determining which numbers occur as smallest eigenvalues of S-matrices. Clearly, this also implies that for every dimension $d$ there are finitely many possible angles $\theta$ for which there are $(d+1) \theta$-equiangular lines in $\mathbf{R}^{d}$. A dictionary listing all eigenvalues of the various switching classes of all graphs of order up to 9 can be found in [BMS]. A peek in this dictionary reveals numerous patterns that suggest generalizations to graphs with more vertices and can be a source of interesting questions. For instance, the smallest angle always seems to correspond to the single edge graph of order $d+1$ and for graphs of order 8 and 9 there is one angle smaller than $\arccos \left(\frac{1}{3}\right)$ and it corresponds to the graph with a single edge. It turns out to be the case for all integers $n$. This was also noted by Seidel and Tsaranov [ST] but they did not include a proof. 


\section{Definitions and Notation}

We denote by $\mathbf{j}$ the vector $(1, \ldots, 1)$ and by $J_{n, m}$ the $n \times m$ matrix with all entries $=1$. The adjacency matrix of a graph $G$ is denoted by $A$ and its S-matrix by $S$. The eigenvalues of $G$ are the eigenvalues of $A$ and its S-eigenvalues are the eigenvalues of $S$. We denote the eigenvalues of $A$ by $\left\{\lambda_{1} \geq \lambda_{2} \geq \cdots \geq \lambda_{n}\right\}$ and of $S$ by $\left\{\sigma_{1} \geq \sigma_{2} \geq \cdots \geq \sigma_{n}\right\}$. The vector $\left(a_{\left(k_{1}\right)}, b_{\left(k_{2}\right)}, \ldots\right)$ denotes a vector where the first $k_{1}$ coordinates are $a$, the next $k_{2}$ coordinates are $b$, etc. The (disjoint) sum of graphs $X+Y$ is the graph with vertex set $V(X) \cup V(Y)$ and edge set $E(X) \cup E(Y)$, where the unions are disjoint. $G(n, k)$ denotes a $k$-regular graph of order $n$ and $\sigma_{\min }(S)$ the smallest eigenvalue of $S$.

In Section 3 we briefly deal with extremal angles and derive some general observations. In Section 4 we exhibit families of ubiquitous angles and families of frequent angles. There is a striking difference between "odd" angles and "even" angles. While the "odd" angles $\arccos (1 /(2 k+1))$ are ubiquitous and we can usually find more than $(d+1) \arccos (1 /(2 k+1))$-equiangular lines in $\mathbf{R}^{d}$, the angles $\arccos (1 / 2 k)$ are only potentially frequent and the maximum number of $\arccos (1 / 2 k)$-equiangular lines in $\mathbf{R}^{d}$ never exceeds $d+1$. It is amusing to compare this situation with the Euclidean distance in $\mathbf{R}^{d}$. In this case there are infinitely many points in $\mathbf{R}^{d}$ with mutual distance even while at most $d+2$ points with mutual distance odd [GRS], [R3] (in other words, it is "easier" to be "odd" than "even" in Elliptic geometry while it is exactly the opposite in Euclidean geometry).

As noted above, in order to construct $n$ nondegenerate $\theta$-equiangular lines in $\mathbf{R}^{d}$ it is enough to construct a graph $G$ of order $n$, with S-matrix $A$, such that $\sigma_{\min }(A)=$ $-1 / \alpha$, where $\alpha=\cos \theta$. The gist of our proofs thus reduces to constructing appropriate families of graphs and identifying their smallest eigenvalue. The following observation will be useful for identifying the eigenvalues. If $S$ is an $S$-matrix and its eigenvalues are $\left\{\sigma_{1}, \ldots, \sigma_{n}\right\}$, then

$$
\begin{aligned}
\operatorname{tr}(S) & =\sum_{i=1}^{n} \sigma_{i}=0, \\
\operatorname{tr}\left(S^{2}\right) & =\sum_{i=1}^{n} \sigma_{i}^{2}=n(n-1) .
\end{aligned}
$$

\section{Angles among Equiangular Lines}

A general useful bound for the smallest angle $\theta$, among $n>d$ lines in $\mathbf{R}^{d}$ (not necessarily equiangular), is derived in [R3]:

$$
\cos \theta \geq \sqrt{\frac{n-d}{d(n-1)}} .
$$

This bound, though relatively easy to derive, carries a lot of "punch." It can be used to obtain surprisingly accurate information about the distances and sizes of large equilateral sets in $\mathcal{E}^{d-1}$. For instance, the largest angle is easy to identify. Indeed, from (3) we know 
that the largest angle determined by any $d+1$ lines in $\mathbf{R}^{d}$ cannot exceed $\arccos (1 / d)$ (a fact that was essentially known to Jung [J] already in 1901, although not in our language). The lines connecting the center of the regular simplex in $\mathbf{R}^{d}$ to its vertices determine $(d+1) \arccos (1 / d)$-equiangular lines in $\mathbf{R}^{d}$. The switching class of graphs corresponding to this set of lines includes the complete graph $K_{d+1}$. For the smallest angle we have:

\section{Theorem 3.1.}

(a) In every dimension $d \geq 2$ the smallest angle determined by $d+1$ equiangular lines is the angle corresponding to the single-edge graph on $d+1$ vertices. The angle is

$$
\begin{aligned}
\theta_{d} & =\arccos \left(\frac{d-3+\sqrt{d^{2}+6 d-7}}{6 d-8}\right) \\
& =\arccos \left(\frac{1}{3}+\frac{4}{9 d}+O\left(\frac{1}{d^{2}}\right)\right) .
\end{aligned}
$$

(b) The only sets of $d+1$ equiangular lines in $\mathbf{R}^{d}, d \geq 7$, with angle smaller than $\arccos \left(\frac{1}{3}\right)$ correspond to the single-edge graph of order $d+1$.

Proof. For the switching class of the single-edge graph on $n$ vertices, the characteristic polynomial of the S-matrix (one pair of -1 's and the rest 1's) is

$\operatorname{det}\left[x I_{d}-\left(\begin{array}{rrrr}0 & -1 & & 1 \\ -1 & 0 & & \\ & & \ddots & \\ 1 & & & 0\end{array}\right)\right]=(x-1)^{d-2}(x+1)\left(x^{2}+(d-3) x-(3 d-4)\right)$.

The smallest eigenvalue is $-1 / \alpha$, the negative root of the quadratic, and the angle $\theta_{d}=\arccos \alpha<\arccos \left(\frac{1}{3}\right)$ is given by (4). A glimpse in the dictionary [BMS], where the spectra of all switching classes of graphs on $n \leq 9$ vertices are given, reveals that the single-edge graph indeed produces the smallest angle for all graphs of order $\leq 9$.

We first observe that if $X$ is a graph, $v \in V(X)$, then by switching on the set of neighbors of $v$, we obtain a graph $Y$ in which $v$ is isolated. $Y$ is the unique graph switching equivalent to $X$ with $v$ isolated. It is easy to see that if $G$ is switching equivalent to the single-edge graph and $G$ has an isolated vertex, then either $G=K_{2}+(n-2) K_{1}$ or $G=$ $K_{1}+\mathrm{K}_{1, n-2}$. We note that if $H$ is an induced subgraph of $G$, then $\sigma_{\min }(G) \leq \sigma_{\min }(H)$.

Claim 3.2. Let $G$ be a graph of order $n \geq 8$. If $G$ has an isolated vertex, at least two edges, and $\sigma_{\min }(G)>-3$, then $G=K_{1}+K_{1, n-2}$.

The proof proceeds by induction on $n$ starting at $n=8$. For $n=8$ see the "dictionary" [BMS]. We now assume that our graph $X$ has $n>8$ vertices, $\sigma_{\min }(X)>-3$, an isolated vertex $w$, and at least two edges. The two edges together with the isolated vertex $w$ account for at most five vertices. Let $\{x, y, z\}$ be three vertices so that the induced 
subgraph on $X \backslash\{x, y, z\}$ still has at least two edges and also contains the isolated vertex $w$. Consider the three induced subgraphs on $X \backslash\{x\}, X \backslash\{y\}$, and $X \backslash\{z\}$. For each of these subgraphs on $n-1$ vertices with the isolated vertex $w$, and at least two edges, the smallest eigenvalue is $\geq \sigma_{\min }(X)>-3$. By induction, each must be $\{w\}+K_{1, n-3}$ and this is true only if $X=\{w\}+K_{1, n-2}$.

Lemma 3.3. If an even integer $2 k$ is an eigenvalue of an $S$-matrix $S$, then $S$ is of odd order.

Proof. $\operatorname{det}(S-2 k I)=0$ and in particular $\operatorname{det}(S-2 k I) \equiv 0(\bmod 2) \operatorname{but} \operatorname{det}(S-2 k I) \equiv$ $\operatorname{det}(J-I) \equiv n-1(\bmod 2)$. Hence $n$ must be odd.

Theorem 3.4. If $\left\{\ell_{1}, \ldots \ell_{n}\right\}$ are $n \theta$-equiangular lines in $\mathbf{R}^{d}(n>d)$ with $\cos \theta=$ $1 / 2 k$, then $n=d+1$ and $d \equiv 2 k(\bmod 4)$.

Proof. If $n>d+1$, then we would have an even number $2 m$ of equiangular lines in $\mathbf{R}^{d}$. Since $2 m>d$, we would get an S-matrix of even order with eigenvalue $-2 k$, contradicting Lemma 3.3.

To prove the second claim, we consider the matrix $A=(S+2 k I)$. Let $A_{i, j}(x), i \neq j$, denote the matrix in which the entries $A_{i, j}$ and $A_{j, i}$ are replaced by a variable $x$. We get $\operatorname{det}\left(A_{i, j}(x)\right)=a x^{2}+b x+c$. Since all entries in $A$ are integers, then so are the coefficients $a, b, c$. Since $A$ is symmetric, for every permutation in the expansion of $\operatorname{det}\left(A_{i, j}(x)\right)$ that contributes to the coefficient $b$, its symmetric permutation contributes exactly the same amount to $b$, hence $b=2 d$. We therefore get $\operatorname{det}\left(A_{i, j}(1)\right)-\operatorname{det}\left(A_{i, j}(-1)\right)=4 d \equiv$ $0(\bmod 4)$. Thus if we replace all the -1 's in $A$ by +1 's the value of the determinant modulo 4 will remain unchanged. Hence $\operatorname{det}(A) \equiv \operatorname{det}(J-I+2 k I)(\bmod 4)$. However, $\operatorname{det}(J-I+2 k I)=(2 k+(n-1))(2 k-1)^{n-1}$ and this is congruent to $0(\bmod 4)$ iff $2 k \equiv n-1(\bmod 4)$.

The following observation was noted and extensively used by Seidel [LS]. We include it for its very simple and short derivation.

Corollary 3.5. If $\left\{\ell_{1}, \ldots, \ell_{n}\right\}$ are $n \theta$-equiangular lines in $\mathbf{R}^{d}(n>2 d)$, then $\cos \theta=$ $1 /(2 k+1)$.

Proof. $\quad-1 / \cos \theta$ is an eigenvalue of an S-matrix of order $n$ with multiplicity $n-d>$ $n / 2$. Hence this eigenvalue must be rational and, since all coefficients of the characteristic polynomial are integers with leading coefficient 1, it must be an integer. By Theorem 3.4 it must be an odd integer.

Remarks. Note that Theorem 3.4 implies that if there are $(d+1) \arccos (1 / 2 k)$ equiangular lines in $\mathbf{R}^{d}$ for infinitely many dimensions $d$, then $\arccos (1 / 2 k)$ would be a frequent angle but not ubiquitous. As for the number of equiangular lines, Corollary 3.5 together with (3) give us key information about the possible angles. If the number of lines $n \geq 2 d+1$ we get that the only possible angles $\theta$ for $n \theta$-equiangular lines in $\mathbf{R}^{d}$ 
are $\theta=\arccos (1 /(2 k+1))$ where $\frac{1}{3} \geq 1 /(2 k+1) \geq \sqrt{(d+1) / 2 d^{2}}$. Thus for instance in $\mathbf{R}^{14}$ (the first dimension for which $v(d)$ is still not known), the only possible angles for 29 equiangular lines are $\arccos \frac{1}{3}$ and $\arccos \frac{1}{5}$. (In searching for $v(14)$, $\arccos \frac{1}{3}$ can be excluded, see [LS].)

Usually, it is relatively simple to construct a graph whose $\mathrm{S}$-matrix has $-1 / \alpha$ as an eigenvalue for given values $\alpha=\cos \theta$. The tricky part is to ascertain that it is the smallest. The relation $S=J-2 A-I$ between the $S$-matrix of $G$ and its adjacency matrix $A$ can be helpful. When $G$ is an $r$-regular graph of order $n$ we have a very tight connection between the eigenvalues of $G$ and $S(G)$. Indeed, since $\mathbf{j}$ is an eigenvector of $A$ and every other eigenvector is orthogonal to $\mathbf{j}$, it follows that if $\left\{r=\lambda_{1} \geq \lambda_{2} \geq \cdots \geq \lambda_{n}\right\}$ are the eigenvalues of $A$, then the eigenvalues of $S$ are $\left\{n-2 r-1,-2 \lambda_{2}-1, \leq-2 \lambda_{3}-1 \leq\right.$ $\left.\cdots \leq-2 \lambda_{n}-1\right\}$. Thus the smallest eigenvalue of $S(G)$ will be $\min \left\{n-2 r-1,-2 \lambda_{2}-1\right\}$. Unfortunately the parameter $\lambda_{2}$ is notoriously difficult to control and even more difficult to keep constant for large families of regular graphs, so its use for constructing ubiquitous or frequent angles may look doubtful. Nevertheless, there are ways to exploit this relation as we see in the next section.

\section{Constructing Ubiquitous and Frequent Angles}

A quick glimpse in the dictionary [BMS] reveals that the angle $\arccos \frac{1}{3}$ appears in every dimension $\geq 3$, in a multitude of nonisometric configurations, and frequently in substantially more than just $d+1$ equiangular lines in $\mathbf{R}^{d}$. We show that the angles $\arccos (1 /(2 k+1))$ behave similarly. As noted before, the situation for the angles $\arccos (1 / 2 k)$ is drastically different. First they are never ubiquitous (but definitely frequent for $k \geq 4$ and "highly" suspicious to be frequent for all $k \geq 2$ ) and second when they occur in $\mathbf{R}^{d}$ the number of lines cannot exceed $d+1$.

Theorem 4.1. Forall $k \geq 1$ and all $d \geq 2 k+1, \mathbf{R}^{d}$ contains $(d+1) \arccos (1 /(2 k+1))$ equiangular lines.

Proof. Let $S(k, i)$ be the S-matrix of the graph $2 K_{k+1}+i K_{1}$. It is a matrix of order $n=2 k+2+i$ :

$$
S(k, i)=\left(\begin{array}{ccc}
-J_{k+1}+I_{k+1} & J_{k+1} & J_{k+1, i} \\
J_{k+1} & -J_{k+1}+I_{k+1} & J_{k+1, i} \\
J_{i, k+1} & J_{i, k+1} & J_{i}-I_{i}
\end{array}\right) .
$$

We first note that, for all $i \geq 0,-(2 k+1)$ is an eigenvalue of $S(k, i)$. Indeed, if we add the first $k+1$ rows of $S+(2 k+1) I$ we get the vector $(k+1) \mathbf{j}$ and the same thing happens when we add rows $k+2 \cdots 2 k+2$.

We now have to show that it is a simple eigenvalue and also the smallest. In $S(k, i)+I$ the bottom $i$ rows are identical and hence $(-1)$ is an eigenvalue of multiplicity $\geq i-1$ (we assume here that $i \geq 1$ ). The first $k+1$ rows in $S(k, i)-I$ are identical and so are the next $k+1$ rows. Hence $\operatorname{rank}(S(k, i)-I) \leq i+2$ and therefore 1 is an eigenvalue with multiplicity $2 k$. Thus far we identified a total of $2 k+i$ eigenvalues: 
$\left\{(1)^{(2 k-i)},(-1)^{(i-1)},-(2 k+1)\right\}$. To identify the remaining two eigenvalues we use the traces of $S(k, i)$ and $S^{2}(k, i)$. Let $\alpha, \beta$ denote the two missing eigenvalues. From $\operatorname{tr}(S(k, i))=0$ and $\operatorname{tr}\left(S^{2}(k, i)\right)=(2 k+i+2)(2 k+i+1)$ we get

$$
\alpha+\beta=i
$$

and

$$
\begin{aligned}
\alpha^{2}+\beta^{2} & =(2 k+i+2)(2 k+i+1)-(2 k+1)^{2}-2 k(i-1) \\
& =4 k i+i^{2}+2 i+2
\end{aligned}
$$

Hence $\{\alpha, \beta\}$ are the roots of the quadratic equation

$$
x^{2}-i x-(2 k i+i+1)=0 .
$$

The smaller root of this quadratic is

$$
\frac{i-\sqrt{i^{2}+4(2 k i+i+1)}}{2}>-(2 k+1) .
$$

Babai asked whether there are other ubiquitous or frequent angles. For such angles the cosine must be irrational. The following theorem identifies such angles.

Theorem 4.2. For all $k \geq 1$ and all $d \geq 2 k, R^{d}$ contains $(d+1) \arccos (1 /(1+2 \sqrt{k}))$ equiangular lines.

Proof. We need to construct S-matrices of order $n \geq 2 k+2$ for which $-1-2 \sqrt{k}$ is the smallest eigenvalue. Let $G_{t}=2 K_{1, k}+t K_{1}$ (that is, $G_{t}$ consists of two disjoint copies of $K_{1, k}$ and $t$ isolated vertices). The S-matrix $S_{t}$ of $G_{t}$ is a square matrix of order $(2 k+2+t)$ that looks as follows:

$$
S_{t}=\left(\begin{array}{ccc}
S S t_{k} & J_{k+1} & J_{k+1, t} \\
J_{k+1} & S S t_{k} & J_{k+1, t} \\
J_{t, k+1} & J_{t, k+1} & J_{t}-I_{t}
\end{array}\right),
$$

where $S S t_{k}$ is the S-matrix of the graph $K_{1, k}$.

The S-matrix $S_{0}$ is simple to handle. Indeed, $S_{0}+I$ has rank 4 , hence -1 is an eigenvalue with multiplicity $2 k-2+t$. In $S_{0}-I$ the first row is $(-1)$ times the $(k+2)$ nd row, so 1 is also an eigenvalue. $v_{0}=\left(1,(1 / \sqrt{k})_{(k)},-1,(-1 / \sqrt{k})_{(k)}\right)$ is an eigenvector with corresponding eigenvalue $-1-2 \sqrt{k}$. Its algebraic conjugate $-1+2 \sqrt{k}$ is also an eigenvalue (this can actually be verified directly; $\left(1,(-1 / \sqrt{k})_{(k)},-1,(1 / \sqrt{k})_{(k)}\right)$ is the corresponding eigenvector). $\operatorname{tr}\left(S_{0}\right)=0$ implies that the remaining eigenvalue is 3 . Thus the eigenvalues of $S_{0}$ are

$$
\left\{-1-2 \sqrt{k},-1^{(2 k-2)}, 1,-1+2 \sqrt{k}, 3\right\} .
$$

For $S_{t}, t \geq 1$, we first identify all but three of its eigenvalues. Note that $S_{t}+I$ has rank 5 and hence -1 is an eigevalue of multiplicity $2 k+2+t-5=2 k+t-3$. A 
simple check as above shows that the vector $v_{0}=\left(1,(1 / \sqrt{k})_{(k)},-1,(-1 / \sqrt{k})_{(k)}, 0_{(t)}\right)$ is an eigenvector with corresponding eigenvalue $-1-2 \sqrt{k}$ and its algebraic conjugate $-1+2 \sqrt{k}$ is also an eigenvalue.

The following eigenvectors correspond to the eigenvalue -1 :

$$
\left(0, x_{1}, \ldots, x_{k}, 0_{(k+1+t)}\right),\left(0_{(k+2)}, x_{1}, \ldots, x_{k}, 0_{(t)}\right),\left(0_{(2 k+2)}, y_{1}, \ldots, y_{t}\right),
$$

where $\sum_{i=1}^{k} x_{i}=0$ and $\sum_{i=1}^{t} y_{i}=0$.

Since the remaining five eigenvectors are orthogonal to these, they must have the form

$$
\left(x, \alpha_{(k)}, y, \beta_{(k)}, \gamma_{(t)}\right) .
$$

When $\gamma=0$ we obtain the two vectors corresponding to the eigenvalues $-1 \pm 2 \sqrt{k}$. We attempt to find the remaining three eigenvalues among the above vectors with $\gamma \neq 0$. Without loss of generality, we may assume that $\gamma=1$. Let $\sigma$ denote an additional eigenvalue corresponding to such an eigenvector. By multiplying the bottom row of $S_{t}$ by $\mathbf{u}=\left(x, \alpha_{(k)}, y, \beta_{(k)}, 1_{(t)}\right)^{\text {tr }}$ we get

$$
x+k \alpha+y+k \beta+(t-1)=\sigma .
$$

Similarly, by multiplying $\mathbf{u}$ by rows $1, k+1,2$, and $k+2$, respectively, we get

$$
\begin{aligned}
-k \alpha+y+k \beta+t & =\sigma x, \\
x+k \alpha-k \beta+t & =\sigma y, \\
-x+(k-1) \alpha+y+k \beta+t & =\sigma \alpha, \\
x+k \alpha-y+(k-1) \beta+t & =\sigma \beta .
\end{aligned}
$$

By adding (6) and (7), we get

$$
x+y+2 t=\sigma(x+y) \Rightarrow x+y=\frac{2 t}{\sigma-1},
$$

and by adding (8) and (9), we get

$$
(2 k-1)(\alpha+\beta)+2 t=\sigma(\alpha+\beta) \quad \Rightarrow \quad \alpha+\beta=\frac{2 t}{\sigma-(2 k-1)} .
$$

Substituting these values in (5) yields

$$
\sigma=\frac{2 t}{\sigma-1}+\frac{2 k t}{\sigma-(2 k-1)}+(t-1)
$$

Simplifying we get the additional eigenvalues as the roots of the cubic:

$q(\sigma)=\sigma(\sigma-1)(\sigma-2 k+1)-2 t(\sigma-2 k+1)-2 k t(\sigma-1)+(t-1)(\sigma-1)(\sigma-2 k+1)$.

Clearly, every root of $q(\sigma)$ is an eigenvalue. We have

$$
q(1)=2 t(2 k-2)>0 ; \quad q(2 k-1)=-2 k t(2 k-2)<0,
$$


hence $q(\sigma)$ has one root in the interval $(1,2 k-1)$ and one root greater than $2 k-1$. To identify the location of the third root we evaluate $q(-1-2 \sqrt{k})$. If it turns out that it is negative it means that the final missing root is greater than $-1-2 \sqrt{k}$. Indeed, we have

$$
\begin{aligned}
& q(-1-2 \sqrt{k}) \\
& =(-2 \sqrt{k}-t) 4 \sqrt{k}(1+\sqrt{k})^{2}-2(k+1) t(-1-2 \sqrt{k})+6 k t-2 t \\
& =-8 k(1+\sqrt{k})^{2}<0 .
\end{aligned}
$$

Theorem 4.3. If $G=G(2 n, 2 k-1)$ is a $(2 k-1)$-regular graph of order $2 n$ and $\lambda_{2}(G)<(2 k-1) / 2$, then there are $4 n-(2 k-1)$ arccos $(1 / 2 k)$-equiangular lines in $\mathbf{R}^{4 n-2 k}$.

Proof. The proof will be accomplished by showing that the smallest S-eigenvalue of the graph $G+(2 n-2 k+1) K_{1}$ is $-2 k$. Let $S$ be the S-matrix of this graph. We assume that the graph is labeled so that the vertices of the initial graph $G$ are labeled $\{1, \ldots, 2 n\}$. If we add the first $2 n$ rows in $S+2 k I$ we get the vector $\left((2 n-2 k+1)_{(2 n)}, 2 n_{(2 n-2 k+1)}\right)$. We get the same vector when we add the bottom $(2 n-2 k+1)$ rows. Hence $-2 k$ is an eigenvalue. To show that it is the smallest, we note that in $S+I$ the bottom $2 n-2 k+1$ rows are all equal to $\mathbf{j}$ and therefore -1 is an eigenvalue with multiplicity $2 n-2 k$. Let $\left\{2 k-1=\lambda_{1} \geq \lambda_{2} \geq \cdots \geq \lambda_{2 n}\right\}$ be the eigenvalues of $G$. Recall that for the eigenvectors $v_{i}, i \geq 2$, we have $\left\langle\mathbf{j}, v_{i}\right\rangle=0$. Hence for the vectors $u_{i}=\left(v_{i}, 0_{(2 n-2 k+1)}\right)$ we have $S u_{i}=\left(\left(-2 \lambda_{i}-1\right) v_{i}, 0_{(2 n-2 k+1)}\right)$. In other words, $\left\{\left(-2 \lambda_{2}-1\right), \ldots,\left(-2 \lambda_{2 n}-1\right)\right\}$ are all eigenvalues of $S$. Note that the smallest eigenvalue among these is $\left(-2 \lambda_{2}-1\right)>-2 k$ by our assumptions. Thus far we identified $4 n-2 k$ eigenvalues. We know that $\sum_{i=1}^{2 n} \lambda_{i}=0$ and hence $\sum_{i=2}^{2 n} \lambda_{i}=-2 k+1$ and therefore the sum of the $(2 n-2 k)$ eigenvalues we identified is $4 k-4 n-1$ and this is negative since $2 n>2 k-1$ and thus the remaining eigenvalue is positive.

Theorem 4.4. If $G(n, k)$ is a $k$-regular graph of order $n, n+k \equiv 1(\bmod 2)$ and $\lambda_{2}(G)<k-\frac{1}{2}$, then there are $4 n-1(\arccos (1 / 2 k))$-equiangular lines in $\mathbf{R}^{4 n-2}$.

Proof. Let $H(n-1, k-1)$ be any $(k-1)$-regular graph of order $n-1$. Note that such a graph always exists. We claim that the smallest S-eigenvalue of the graph $G+H$ is $-2 k$. Let $S$ be the S-matrix of this graph. We first note that if we add the top $n$ rows of $S+2 k I$ we get the vector $\left((n-1)_{(n)}, n_{(n-1)}\right)$ and we also get the same vector when we add the bottom $(n-1)$ rows. Again, using the regularity of the matrices $G(n, k)$ and $H(n-1, k-1)$ it can be verified along the same lines as in the proof of Theorem 4.3 that $-2 k$ is the smallest eigenvalue. We leave the details to the reader.

Our first attempt to identify frequent angles was for $k=2$. We found the angle $\arccos \frac{1}{4}$ to be very elusive. Using Theorem 4.3 did not go very far but did produce a mild surprise. The graphs $K_{4}, K_{3,3}, 3$-Cube, Peterson, and the cubic 6-cage (14 vertices) have $\lambda_{2}=-1,0,1,1$, and $\sqrt{2}$, respectively. All are less than $\frac{3}{2}$ and thus produce 5,9 , 13,17 , and $25\left(\arccos \frac{1}{4}\right)$-equiangular lines in $\mathbf{R}^{d}$ for $d=4,8,12,16$, and 24 . Twentyone lines in $\mathbf{R}^{20}$ are missing. To our surprise we discovered that $\lambda_{2}(G(12,3))>\frac{3}{2}$. 
Brendan McKay's list of cubic graphs helped us verify this lack of monotonicity in the sequence $\min \left\{\lambda_{2}(G(2 n, 3))\right\}$. With Brendan's kind help and powerful graph programs, we managed to construct some short sequences of graphs $G$ for which $\sigma_{\min }(G)=-4$. These lists include:

$$
\begin{aligned}
P_{4}+i K_{2}+(45-6 i) K_{1} & (0 \leq i \leq 7), \\
C_{8}+i K_{2}+(21-6 i) K_{1} & (0 \leq i \leq 3), \\
C_{6}+i K_{2}+(21-6 i) K_{1} & (0 \leq i \leq 2), \\
K_{1,3}+i K_{2}+(21-6 i) K_{1} & (0 \leq i \leq 3), \\
C_{7}+i K_{2}+(18-6 i) K_{1} & (0 \leq i \leq 3) .
\end{aligned}
$$

These short sequences produce graphs of orders $4 k+1$ for $3 \leq k \leq 12$. A curious phenomenon is evident: one copy of $K_{2}$ seems to "balance" six isolated vertices. Indeed, this can be proved in a much more general setting albeit it affects the smallest eigenvalue. So far we have not been able to extend our constructions but we firmly believe that Smatrices of orders $4 k+1$ with $\sigma_{\min }(S)=-4$ exist for all integers $k$. Theorems 4.3 and 4.4 can be used to produce frequent angles. From Theorem 3.4 it is enough to show that in infinitely many dimensions $d$ there are $(d+1) \arccos (1 / 2 k)$-equiangular lines in $\mathbf{R}^{d}$. Expanders can be used for this purpose. Alon [A] showed that $\underline{\lim } \lambda_{2}(G(n, k))=$ $2 \sqrt{k-1}$. Hence for $k \geq 4$ one can hope to find infinitely many regular graphs $G=$ $G(n, k)$ for which $\lambda_{2}(G)<k-\frac{1}{2}$. We conjecture that $\forall n \geq 5, \exists G=G(n, 4)$ with $\lambda_{2}<$ $\frac{7}{2}$. This of course will prove that for all integers $d=4 m, m \geq 2$, there are $d+1\left(\arccos \frac{1}{8}\right)$ equiangular lines in $\mathbf{R}^{d}$. Existence of infinitely many regular graphs $G(n, r)$ for which $\lambda_{2}(G(n, r))<r / 2$ (even for fixed $r$ ) can be deduced by the probabilistic method, and thus prove that frequent angles exist. Constructive methods for regular graphs which are good expanders were found by Lubotzky et al. [LPS]. They construct the Ramanujan graphs $G\left(q\left(q^{2}-1\right), p+1\right)$, that is graphs satisfying $\lambda_{2}\left(G\left(q\left(q^{2}-1\right), p+1\right)\right) \leq 2 \sqrt{p}$ for $p, q$ distinct primes of the form $4 k+1$. Though these graphs do not satisfy the condition of Theorem 4.4, by taking the prism over each such graph we obtain a graph $G\left(2 q\left(q^{2}-1\right), p+2\right)$ for which $\lambda_{2}<p+\frac{3}{2}$ thus yielding infinitely many frequent angles that are not ubiquitous. These observations led us to:

Conjecture 4.5. For all $k \geq 2$ and $d \geq 0$ there are $(2 k+4 d+1) \arccos (1 / 2 k)$ equiangular lines in $\mathbf{R}^{2 k+4 d}$.

Conjecture 4.6. For all $r \geq 4$ and all $n>r, n r \equiv 2(\bmod 4), \exists G(n, r)$ with $\sigma_{\min }(G(n, r))<r-\frac{1}{2}$.

\section{Acknowledgment}

We thank Brendan McKay for his help in identifying many of the graphs $G$ with $\sigma_{\min }(G)=-4$ and the referee for many helpful remarks. 


\section{References}

[AZ] M. Aigner and G. M. Ziegler, Proofs from THE BOOK, Springer-Verlag, New York, 1998, pp. 83-88.

[A] N. Alon, Eigenvalues and expanders, Combinatorica, 6 (1986), 83-96.

[B] L. M. Blumenthal, Theory and Applications of Distance Geometry, Clarenden Press, Oxford, 1953.

[BMS] F. C. Bussemaker, R. Mathon, and J. J. Seidel, Tables of Two-Graphs, Report 79-WSK-05, Technological University Eindhoven, 1979.

[GRS] R. L. Graham, B. L. Rothschild, and E. G. Strauss, Are there $n+2$ points in $E^{n}$ with odd integral distances?, Amer. Math. Monthly, 81 (1974), 21-25.

[HS] J. Haantjes and J. J. Seidel, The congruence order of the elliptic plane, Proc. Kon. Ned.Akad. Wetensch., A 50 (1947), 892-894.

[J] H. W. E. Jung, Über die klienste Kugel, die eine räumliche Figur einschließt, J. Reine Angew. Math., 123 (1901), 241-257.

[KK] J. Kahn and G. Kalai, A counterexample to Borsuk's conjecture, Bull. Amer. Math. Soc., 29 (1993), 60-62.

[LS] P. W. H. Lemmens and J. J. Seidel, Equiangular lines, J. Algebra, 24 (1973), 494-512.

[LPS] A. Lubotzky, R. Philips, and P. Sarnak, Ramanujan graphs, Combinatorica, 8 (1988), 261-277.

[R1] R. Robinson, Solution to problem no. 10293, Amer. Math. Monthly, 102 (1995), 278-279.

[R2] M. Rosenfeld, Problem no. 10293, Amer. Math. Monthly, 100 (1993), 291.

[R3] M. Rosenfeld, In praise of the Gram matrix, in The Mathematics of Paul Erdös, R. L. Graham and J. Nešetřil, eds., Springer-Verlag, New York, 1997, pp. 318-323.

[S] J. J. Seidel, A survey of two-graphs, Proc. Internat. Colloq. Teorie Combinatorie (Roma, 1973), Acad. Naz. Lincei, Roma, 1976, pp. 481-511.

[ST] J. J. Seidel and S. V. Tsaranov, Two-graphs, related groups, and Root systems, Bull. Soc. Belg., XLII (1990), 695-711.

[vLS] J. H. van Lint and J. J. Seidel, Equilateral point sets in elliptic geometry, Nederl. Akad. Wetersch. Indag. Math., 28 (1966), 335-348.

Received March 31, 1999, and in revised form August 12, 1999. Online publication May 8, 2000. 